\title{
Placebo expectancy effects in the relationship between glucose and cognition
}

\author{
Michael W. Green ${ }^{1}$, Moira A. Taylor ${ }^{2}$, Nicola A. Elliman ${ }^{1}$ and Orli Rhodes ${ }^{2}$ \\ ${ }^{1}$ Institute of Food Research, Whiteknights Road, Earley Gate, Reading, Berkshire, RG6 6BZ, UK \\ ${ }^{2}$ Dept of Human Nutrition, Kings College London, Franklin-Wilkins Building, 150 Stamford Street, London SE1 8WA, UK
}

(Received 25 October 2000 - Revised 22 January 2001 - Accepted 4 April 2001)

\begin{abstract}
The present study investigated the extent of expectancy in the ability of glucose to affect cognitive performance. Using a within-subjects design, subjects $(n$ 26) completed four experimental sessions (in counterbalanced order and after an initial practice session) during which they were given a $500 \mathrm{ml}$ drink 30 min prior to completing a cognitive assessment battery. In addition, all subjects completed a baseline practice session during which they were given no drink. During two of the sessions, subjects were given a drink containing $50 \mathrm{~g}$ glucose and on the other two they were given a drink containing aspartame. A balanced placebo design was used, such that for half the sessions subjects were accurately informed as to the content of the drink (glucose or aspartame), whereas in the other two sessions they were misinformed as to the content of the drink. The task battery comprised a 6 min visual analogue of the Bakan vigilance task, an immediate verbal free-recall task, an immediate verbal recognition memory task and a measure of motor speed (two-finger tapping). Blood glucose and self-reported mood were also recorded at several time points during each session. Glucose administration was found to improve recognition memory times, in direct contrast to previous findings in the literature. Glucose administration also improved performance on the Bakan task (relative to the control drink), but only in sessions where subjects were informed that they would receive glucose and not when they were told that they would receive aspartame. There were no effects either of the nature of the drink or expectancy on the other measures. These results are interpreted in terms of there being some contribution of expectancy concerning the positive effects of glucose on cognition in studies which have not used an equi-sweet dose of aspartame as a control drink.
\end{abstract}

Glucose: Cognition: Placebo effect

There is currently a great deal of research interest in the degree to which cognitive processing efficiency is affected by nutritional variables. One nutritional variable which has been the focus of a large body of this research interest is glucose. Given that glucose is the main source of fuel for the brain (e.g. Sieber \& Traystmen, 1992) under normal circumstances, it would seem to be a reasonable hypothesis that alterations in glucose intake will impact upon brain function (Benton \& Sargent, 1992). There are two strands of evidence upon which this hypothesis is based.

First, there is a body of evidence (albeit inconsistent) indicating that relatively short periods of food deprivation, such as missing individual meals, are associated with impairments in cognitive processing efficiency. For instance, whilst it has been shown that missing breakfast impairs performance on some measures (Pollitt et al. 1983, 1996), such brief periods of fasting have also been found to improve performance on others (Pollitt et al. 1981; Green et al. 1997). Further, it has been found that brief periods of fasting produces no significant deficit in performance (Green et al. 1995). In addition, it has been demonstrated that food deprivation-induced drops in blood glucose levels are not significantly associated with impaired performance (Green et al. 1997).

The second strand of evidence which bears relevance to the hypothesis that manipulating blood glucose levels will directly impact upon brain function is that of the work examining the effects of glucose administration upon cognition. A number of studies have shown that administration of a glucose drink results in improved performance on a number of measures of cognitive processing efficiency (e.g. Manning et al. 1990; Benton \& Owens, 1993; Foster et al. 1998). A previous review of the literature up to 1994, however (Rogers \& Lloyd, 1994),

* Corresponding author: Dr Michael W. Green, present address Psychology Group, School of Life and Health Sciences, Aston University, Aston Triangle, Birmingham B4 7ET, UK, fax +44 121359 3257, email m.w.green@aston.ac.uk 
concluded that the results of these studies were equivocal and that there was little convincing or consistent evidence for a beneficial effect of glucose administration upon the cognitive function of healthy subjects.

More recent work in this area has centered on the hypothesis that glucose administration will selectively enhance memory function (e.g. Gold, 1986). There is, however, some question as to the wider applicability of this work, since it has been specifically demonstrated that glucose administration improves memory function in the elderly, but not in young adult populations (Manning et al. 1997). Even in healthy young adults, the effects appear to be somewhat task and study specific in nature. For instance, whilst glucose administration improved verbal recall in some studies (Foster et al. 1998), other studies have found no beneficial effects of glucose on immediate verbal recall (Benton \& Owens, 1993; Benton et al. 1994).

A number of these studies have also found correlations between task performance and blood glucose levels, either in addition to (Brooke \& Toogoode, 1973; Hall et al. 1989; Manning et al. 1990; Foster et al. 1998), or in the absence of (Benton \& Owens, 1993; Benton et al. 1994), significant treatment effects. According to Rogers \& Lloyd (1994), these significant correlations in the absence of treatment effects suggest that the relationships between glucose and cognition in the healthy population may be epiphenomenal rather than direct. For instance, it is suggested that high baseline or rapidly rising levels of blood glucose in these studies reflected increased sympathetic activity, including an increase of cortisol and adrenaline. It was hypothesised, therefore, that cortisol and adrenaline were the main variables mediating task performance in these studies. Although the physiological mechanisms underlying the relationships between arousal and performance (e.g. Johnson et al. 1992) may include mechanisms such as increased glucose uptake and utilisation by the central nervous system, there is no evidence to suggest that this is the mechanism underlying stress or glucose administration related improvements in performance. It has been found, however, that, whilst manipulating arousal levels by means of incentive motivation (Green et al. 1997) does produce selective improvements in performance, these are unrelated to glucose levels.

A majority of these studies have either used saccharin (e.g. Benton et al. 1987; Hall et al. 1989; Manning et al. 1997), a non-sweet drink (e.g. Brooke \& Toogoode, 1973) or nothing at all (e.g. Lapp, 1981) as a control condition. Although a number of these studies have matched the glucose and saccharin drinks for sweetness on the basis of pilot testing (e.g. Hall et al. 1989; Parsons \& Gold, 1992), a number have not (e.g. Messier et al. 1999). Certainly, none has investigated whether subjects could actually detect a difference between the glucose and placebo drinks. Since saccharin and glucose have differences in taste, it is possible that subjects were able to discern which of the two drinks actually contained glucose and which contained saccharin. It is possible, therefore, there was some element of expectancy active in the studies which have used saccharin or a non-sweet drink as a control. This may be less true of the studies which use aspartame as a placebo control since there are fewer detectable differences in taste between glucose and aspartame (e.g. Benton \& Owens, 1993).
There is evidence from the fields of alcohol and caffeine research which indicates that expectancy concerning the nature of the substance given exerts a strong influence upon task performance (e.g. Kirsch \& Weixel, 1988; Peterson et al. 1990; Fillmore et al. 1998). This relationship is, however, not straightforward. For instance, it has been found that expectancy effects upon performance only occur within the active treatment condition (Finnigan et al. 1995) and that only subjectively perceived ability to perform is affected (Hammersley et al. 1998). Further, expectancy effects are dependent on the dose of the active substance (Finnigan et al. 1995) and that the nature of the effect differs according to the active substance used (Fillmore et al. 1994). The evidence concerning positive expectancy effects (expecting positive effects from a substance, whether it be the active substance or placebo) is somewhat more mixed. Whilst improvements in task performance have been demonstrated as a result of positive expectancy (Fillmore \& Vogel-Sprott, 1992), this pattern of results has not been uniformly replicated. For instance, Kvavilashvili \& Ellis (1999) found that positive expectancies regarding a placebo did not affect performance on a delayed free-recall task whereas negative expectancies were associated with poorer recall, although self-reported performance did yield expectancy congruent effects. This was explained in terms of 'reverse placebo effects' whereby subjects in the positive placebo group exert less effort on task performance due to the fact that they believe the treatment will enhance performance automatically.

The purpose of the present study, therefore, was to investigate the extent to which the beneficial effects of glucose on cognitive function are mediated by expectancy when aspartame is used as a placebo control. Aspartame was used as the placebo control substance in the present study to ensure that any observed expectancy effects could be accounted for in terms of experimental instructions, rather than perceived differences in taste between the two experimental drinks

\section{Methods and materials}

\section{Overview}

After an initial baseline test session, subjects completed four experimental test sessions in which their cognitive performance was assessed. On two of these sessions, they ingested a glucose drink prior to testing and on the other two they ingested a non-glucose-laden placebo drink. In one of each drink conditions, subjects were accurately informed as to the nature of the drink prior to testing, and in the other they were informed that they had been given the other drink.

\section{Subjects}

The subjects comprised male and female undergraduate students from King's College, London, UK, and their associates ( $n$ 26). All subjects were aged between 18 and 40 years. Prior to recruitment, all subjects were informed as to the nature of the experimental procedures which they would undergo. Subjects with a history of diabetes and phenylketonuria were excluded, as were subjects who were 
currently dieting to lose weight. The study was approved by the King's College research ethics committee and all subjects gave informed consent prior to participation. Each subject was paid $£ 10$ per test session for their participation in the study.

\section{Design and procedure}

Using a repeated-measures design, each subject was tested over five sessions. After an initial practice session in which subjects completed a cognitive assessment battery and mood scales, and in which baseline blood glucose levels were measured, they then completed four sessions in which they again completed these measures $30 \mathrm{~min}$ after consuming a $500 \mathrm{ml}$ drink. On two sessions, the drink contained glucose and on two sessions, the drink contained aspartame (placebo). On one of the two sessions during which subjects received either glucose or aspartame, they were correctly informed as to the nature of the drink they would receive and on the other they were told that they would receive the other drink. Each subject was tested individually, in a session lasting roughly $1 \mathrm{~h}$. The testing environment was identical for all subjects. At the time of testing, each subject had abstained from food, glucose- or sucrose-containing drinks and strenuous exercise for a period of $8 \mathrm{~h}$. All test sessions took place between 09.00 and 12.00 hours.

At the start of each session, subjects were given information (either accurate or false) concerning the drink they were about to consume. They then completed a set of $100 \mathrm{~mm}$ visual analogue mood scales, anchored at each end with the statements 'Not at all' to 'Extremely'. The moods rated were 'Friendly', 'Jittery', 'Headache', 'Drowsy', 'Cheerful', 'Calm', 'Uncertain', 'Dejected', 'Energetic', 'Placid', 'Confident', 'Tired', 'Anxious' and 'Lively'. Following completion of this first set of mood ratings, a measure of blood glucose was obtained, using a fingerprick lancet. Glucose levels were assayed using an Accutrend GC meter (measuring range $1.1-33.3 \mathrm{mmol} / \mathrm{l}$; Boehringer Mannheim, Lewes, East Sussex, UK). In all but the practice session, subjects then consumed the experimental drink and waited for a period of $30 \mathrm{~min}$. Subjects then completed a computer-based battery of cognitive function measures (with another blood glucose measurement being taken at the mid-point of cognitive assessment). Upon completion of the task battery, subjects completed a second set of mood scales and a third blood glucose measurement was taken.

The glucose drink was formulated with $500 \mathrm{~g}$ glucose, $10 \mathrm{~g}$ citric acid, $5 \mathrm{ml}$ lemon-flavour essence $/ 5$ litres water. This gave a total glucose load of $50 \mathrm{~g} / \mathrm{drink}$, which has previously been used in studies which have shown effects of glucose on cognition (e.g. Benton \& Owens, 1993). The placebo drink was of the same formulation, except that $2 \mathrm{~g}$ aspartame/5 litres water was substituted for the glucose. The amount of aspartame was calculated according to the sweetness scale for sucrose, accounting for the fact that glucose has a sweetness of 0.75 relative to sucrose. Pilot testing indicated that there were no noticeable differences in taste between the two drinks $(P>0 \cdot 05)$.

Cognitive assessment battery. The tasks were presented to subjects via a $486 \mathrm{MHz}$ PC running MEL v. 2 (Psychology Software Tools Inc., Pittsburgh, PA, USA) with a $33 \mathrm{~cm}$ colour monitor. The tasks were presented in the same order during each session and were as follows. (1) Two-finger tapping task: subjects were required to alternately tap the ' 1 ' and ' 2 ' keys of the numeric keypad as quickly as possible, using the index and second fingers of the preferred hand. The dependent variable was the time taken to make 300 key presses. (2) Recognition memory task: this was an adaptation of the Sternberg (1966) recognition memory task. Subjects were presented with two lists of twenty words (in a randomised order), with one list being presented at $1 \mathrm{~s}$ per word and the other at $2 \mathrm{~s}$ per word. Immediately after presentation of each stimulus list, subjects were presented with a probe recognition set of forty words. This comprised the original twenty words from the stimulus list, in addition to twenty filler words. The filler stimuli were matched for length and frequency of occurrence in written English with the test items. Subjects had to decide whether each of the forty words was present in the original list or not by pressing either the ' 1 ' key (labelled 'Present') or the ' 3 ' key (labelled 'Not Present') on the numeric keypad. The dependent variables were the number of correct recognitions made and the total response time to make those decisions. (3) Verbal free-recall task: subjects were presented with two lists of twenty words each, one list being presented at $1 \mathrm{~s}$ per word and the other at $2 \mathrm{~s}$ per word. Immediately after the presentation of each list, subjects were given 4 min to recall as many words as possible from that list. (4) Bakan task: this was a 6 min long visual analogue of the procedure developed by Bakan (1959) in which subjects were presented with a continuous stream of single digits (1-9) which appeared one at a time, in the centre of the visual display unit monitor. The subjects' task was to press a labelled response key, as quickly as possible, when they believed that they had detected a sequence of either three odd numbers or three even numbers occurring consecutively. The stimulus exposure duration was $600 \mathrm{~ms}$, with no inter-stimulus interval and forty-eight potential correct targets. The dependent variable was the number of correct hits made over the course of the task.

\section{Results}

For all analyses, Fisher's least significant difference test (with appropriate correction for multiple comparisons) was used for post-hoc testing. Analyses of task performance were conducted using analysis of covariance, with practice session performance being used as the covariate. Uncorrected mean scores are presented in the following data tables.

\section{Blood glucose levels}

The mean blood glucose levels are shown in Table 1. The raw mean values presented in Table 1 suggest that the data violate the assumption of homogeneity of variance necessary for ANOVA. Actual analyses, therefore, were conducted on a logarithmic transformation of the raw data. Glucose levels were analysed via three-way ANOVA (drink, expectancy and within session time point as factors), with time point included as a repeated measure. There was a significant interaction between the drink and time point 
Table 1. Blood glucose levels $(\mathrm{mmol} / \mathrm{l})$ at the start, mid-point and end of each test session for each of the four experimental conditions*

(Mean values and standard deviations for twenty-six subjects)

\begin{tabular}{|c|c|c|c|c|c|c|c|c|}
\hline & \multicolumn{2}{|c|}{ GP/TP } & \multicolumn{2}{|c|}{ GP/TG } & \multicolumn{2}{|c|}{ GG/TG } & \multicolumn{2}{|c|}{ GG/TP } \\
\hline & Mean & $\overline{S D}$ & Mean & $\overline{\mathrm{SD}}$ & Mean & $\overline{S D}$ & Mean & $\overline{\mathrm{SD}}$ \\
\hline Baseline measurement & 4.5 & 0.6 & $4 \cdot 3$ & $0 \cdot 8$ & $4 \cdot 2$ & 0.6 & $4 \cdot 2$ & 0.4 \\
\hline 2nd measurement & $4 \cdot 2$ & $1 \cdot 1$ & $4 \cdot 2$ & $0 \cdot 8$ & $6 \cdot 7$ & 1.9 & $6 \cdot 5$ & $2 \cdot 0$ \\
\hline Final measurement & $4 \cdot 2$ & $1 \cdot 1$ & $4 \cdot 1$ & 0.9 & $6 \cdot 0$ & 1.6 & $5 \cdot 8$ & $2 \cdot 0$ \\
\hline
\end{tabular}

GP/TP, given placebo/told placebo; GP/TG, given placebo/told glucose; GG/TG, given glucose/told glucose; $\mathrm{GG} / \mathrm{TP}$, given glucose/told placebo.

${ }^{*}$ For details of subjects and procedures, see pp. 174-175.

factors $(F(2,50) 43 \cdot 00, \quad P<0 \cdot 001)$. Post-hoc analyses indicated that the blood glucose levels of those subjects who had received the glucose drink rose significantly from first to second measurement $(P<0 \cdot 01)$. There were also significant main effects of drink $(F(1,25)$ 48.98, $P<0 \cdot 001)$ and within session time point $(F(2,50) 26 \cdot 00$, $P<0 \cdot 001)$. There was no significant effect of expectancy or significant interactions involving expectancy $(P>0 \cdot 05)$.

\section{Task performance}

The mean task performance data are shown in Table 2.

\section{Bakan Task}

The data for the Bakan task (correct hits) were analysed via three-way analysis of covariance (drink, expectancy and trial block as factors), with trial block being included as a repeated measure. There was a significant interaction between drink and expectancy $(F(1,25) 5.94, P=0 \cdot 022)$. Post-hoc testing revealed that when subjects were given glucose and were told that they would receive glucose, they made significantly more correct hits than in any other condition $(P<0 \cdot 01)$. There was also a significant main effect of trial block $(F(5,125) 10.53, P<0.001)$, indicating that subjects made progressively fewer correct hits over the course of the task, in addition to a significant main effect of drink $(F(1,25) 19 \cdot 60, P<0 \cdot 001)$. There were no other significant main effects or interactions $(P>0 \cdot 05)$.

\section{Immediate verbal recall}

The data for this task were analysed via three-way analysis of covariance (drink, expectancy and presentation speed as factors). Overall, subjects correctly recalled more words in the $2 \mathrm{~s}$ per word display condition than the $1 \mathrm{~s}$ per word condition $(F(1,25) 12.77, P<0.005)$. There were no other significant main effects or interactions $(P>0.05)$.

\section{Recognition memory}

This task yielded two outcome measures, these being response times to make recognition decisions and the number of words correctly recognised as being presented in the memorised lists. Both variables were analysed via threeway analysis of covariance (factor structure being identical to that used for the immediate verbal recall task).

Response times. Overall, response times were faster during sessions in which subjects received glucose than when they received placebo $(F(1,25) 8.35, P=0.008)$. In addition, there was a marginally significant trend for subjects to respond more quickly when they were told that

Table 2. Mean cognitive performance scores for all four experimental conditions* (Mean values and standard deviations for twenty-six subjects)

\begin{tabular}{|c|c|c|c|c|c|c|c|c|}
\hline & \multicolumn{2}{|c|}{ GP/TP } & \multicolumn{2}{|c|}{ GP/TG } & \multicolumn{2}{|c|}{ GG/TG } & \multicolumn{2}{|c|}{ GG/TP } \\
\hline & Mean & SD & Mean & SD & Mean & SD & Mean & SD \\
\hline Bakan task (mean correct hits) & $4 \cdot 0$ & $2 \cdot 0$ & $4 \cdot 2$ & $2 \cdot 1$ & $4 \cdot 7$ & 1.8 & $3 \cdot 9$ & 1.9 \\
\hline Recognition memory task & & & & & & & & \\
\hline \multicolumn{9}{|l|}{ Recognition times (ms) } \\
\hline $1 \mathrm{~s}$ presentation & 1021 & 226 & 990 & 179 & 948 & 137 & 942 & 142 \\
\hline $2 \mathrm{~s}$ presentation & 1036 & 209 & 1008 & 184 & 982 & 161 & 946 & 173 \\
\hline \multicolumn{9}{|l|}{ Words correctly recognised } \\
\hline 1s presentation & 33.4 & 3.9 & $32 \cdot 8$ & 3.5 & $32 \cdot 7$ & 4.0 & 33.0 & 2.9 \\
\hline $2 \mathrm{~s}$ presentation & 33.6 & $3 \cdot 2$ & $34 \cdot 8$ & $2 \cdot 6$ & 34.9 & 4.9 & $34 \cdot 1$ & 3.6 \\
\hline \multicolumn{9}{|c|}{ Verbal recall (words correctly recalled) } \\
\hline 1s presentation & $6 \cdot 8$ & $2 \cdot 6$ & $6 \cdot 6$ & $2 \cdot 4$ & $7 \cdot 2$ & $3 \cdot 2$ & $6 \cdot 6$ & $2 \cdot 4$ \\
\hline $2 \mathrm{~s}$ presentation & $7 \cdot 8$ & 2.5 & $7 \cdot 5$ & $2 \cdot 4$ & $8 \cdot 1$ & 2.5 & 8.8 & $2 \cdot 7$ \\
\hline Two-finger tapping (taps/s) & $7 \cdot 0$ & $2 \cdot 3$ & $7 \cdot 1$ & $2 \cdot 2$ & $6 \cdot 9$ & $2 \cdot 3$ & $7 \cdot 1$ & $2 \cdot 3$ \\
\hline
\end{tabular}

GP/TP, given placebo/told placebo; GP/TG, given placebo/told glucose; GG/TG, given glucose/told glucose; GG/TP, given glucose/told placebo.

${ }^{*}$ For details of subjects and procedures, see pp. 174-175. 
they were receiving glucose than when they were told they were receiving placebo $(F(1,25) 3 \cdot 12, P=0 \cdot 089)$. There were no other significant main effects or interactions $(P>0 \cdot 05)$.

Correct recognition decisions. Overall, there were more correct decisions made in the condition where words were initially presented for $2 \mathrm{~s}$ each than when they were presented for $1 \mathrm{~s}$ each $(F(1,25) 7 \cdot 28, P=0 \cdot 012)$. There were no other significant main effects or interactions $(P>0.05)$.

\section{Two-finger tapping task}

The data for this task (number of taps per s) were analysed via three-way analysis of covariance (factor structure identical to that of the analysis of covariance for the Bakan task). There was a significant main effect of trial block $(F(5$,
125) $34 \cdot 12, P<0 \cdot 001$ ), with progressively fewer taps being made over the course of the task. This can be interpreted in terms of a within trial fatigue effect. There were no other significant main effects or interactions $(P>0.05)$.

\section{Mood state}

Each mood scale was analysed separately via three-way ANOVA (drink, expectancy and time point as factors), with time point included as a repeated measure and appropriate Bonferroni corrections for multiple comparisons. The data are shown in Table 3.

There was a significant three-way interaction for ratings of 'Dejected' $(F(1,25) 6 \cdot 65, P=0 \cdot 016)$. Post-hoc analysis revealed baseline differences in ratings of dejection between sessions in which subjects were told they would receive

Table 3. Mean affective state scores $(\mathrm{cm})$ for all four experimental conditions* (Mean values and standard deviations for twenty-six subjects)

\begin{tabular}{|c|c|c|c|c|c|c|c|c|}
\hline & \multicolumn{2}{|c|}{ GP/TP } & \multicolumn{2}{|c|}{ GP/TG } & \multicolumn{2}{|c|}{ GG/TG } & \multicolumn{2}{|c|}{ GG/TP } \\
\hline & Mean & SD & Mean & SD & Mean & SD & Mean & SD \\
\hline \multicolumn{9}{|l|}{ Friendly } \\
\hline 1st measurement & $64 \cdot 8$ & $22 \cdot 8$ & 63.9 & $23 \cdot 3$ & $60 \cdot 7$ & $24 \cdot 6$ & 71.5 & $16 \cdot 7$ \\
\hline 2nd measurement & $65 \cdot 1$ & $22 \cdot 6$ & $63 \cdot 1$ & $22 \cdot 8$ & $66 \cdot 1$ & $23 \cdot 3$ & 69.5 & $18 \cdot 6$ \\
\hline \multicolumn{9}{|l|}{ Jittery } \\
\hline 1st measurement & 24.9 & $24 \cdot 8$ & 23.9 & $22 \cdot 9$ & $21 \cdot 1$ & $23 \cdot 9$ & $20 \cdot 1$ & $21 \cdot 3$ \\
\hline 2nd measurement & $15 \cdot 7$ & $19 \cdot 5$ & 21.5 & $20 \cdot 7$ & 23.5 & $23 \cdot 6$ & $16 \cdot 8$ & 21.9 \\
\hline \multicolumn{9}{|l|}{ Headache } \\
\hline 1st measurement & $13 \cdot 2$ & $23 \cdot 2$ & $15 \cdot 0$ & $23 \cdot 4$ & $17 \cdot 7$ & $26 \cdot 4$ & $10 \cdot 7$ & $17 \cdot 7$ \\
\hline 2nd measurement & $15 \cdot 6$ & $25 \cdot 6$ & $17 \cdot 2$ & $27 \cdot 3$ & $17 \cdot 5$ & 24.9 & $12 \cdot 7$ & $17 \cdot 0$ \\
\hline \multicolumn{9}{|l|}{ Drowsy } \\
\hline 1st measurement & $32 \cdot 7$ & $26 \cdot 7$ & $34 \cdot 8$ & $29 \cdot 0$ & $23 \cdot 9$ & $20 \cdot 2$ & $33 \cdot 1$ & $24 \cdot 4$ \\
\hline 2nd measurement & $24 \cdot 0$ & $22 \cdot 1$ & $30 \cdot 6$ & $27 \cdot 7$ & $27 \cdot 4$ & $25 \cdot 6$ & $31 \cdot 3$ & $26 \cdot 6$ \\
\hline \multicolumn{9}{|l|}{ Cheerful } \\
\hline 1st measurement & $65 \cdot 1$ & $21 \cdot 2$ & $64 \cdot 8$ & $19 \cdot 6$ & 68.4 & $22 \cdot 4$ & $65 \cdot 3$ & $20 \cdot 0$ \\
\hline 2nd measurement & $64 \cdot 9$ & $20 \cdot 2$ & $65 \cdot 3$ & $22 \cdot 2$ & $66 \cdot 9$ & 21.5 & $67 \cdot 5$ & $18 \cdot 9$ \\
\hline \multicolumn{9}{|l|}{ Calm } \\
\hline 1st measurement & $59 \cdot 4$ & $26 \cdot 7$ & $61 \cdot 6$ & $22 \cdot 1$ & $55 \cdot 7$ & $27 \cdot 2$ & $62 \cdot 0$ & $23 \cdot 7$ \\
\hline 2nd measurement & $63 \cdot 7$ & $23 \cdot 9$ & 63.2 & 27.5 & $63 \cdot 3$ & $25 \cdot 1$ & $64 \cdot 3$ & $27 \cdot 9$ \\
\hline \multicolumn{9}{|l|}{ Uncertain } \\
\hline 1st measurement & $15 \cdot 9$ & $16 \cdot 5$ & $18 \cdot 7$ & $22 \cdot 0$ & $19 \cdot 4$ & $19 \cdot 2$ & $26 \cdot 7$ & $24 \cdot 6$ \\
\hline 2nd measurement & $18 \cdot 7$ & $18 \cdot 0$ & $23 \cdot 0$ & $22 \cdot 2$ & 24.9 & $23 \cdot 4$ & 22.5 & $25 \cdot 1$ \\
\hline \multicolumn{9}{|l|}{ Dejected } \\
\hline 1st measurement & $14 \cdot 0$ & $16 \cdot 7$ & $11 \cdot 2$ & $15 \cdot 0$ & 11.9 & $16 \cdot 9$ & 17.5 & $23 \cdot 6$ \\
\hline 2nd measurement & $10 \cdot 5$ & $14 \cdot 6$ & 13.5 & $16 \cdot 5$ & $15 \cdot 9$ & $23 \cdot 5$ & $13 \cdot 6$ & $21 \cdot 2$ \\
\hline \multicolumn{9}{|l|}{ Energetic } \\
\hline 1st measurement & 44.9 & $27 \cdot 9$ & $42 \cdot 6$ & $28 \cdot 0$ & $43 \cdot 9$ & $27 \cdot 6$ & $45 \cdot 2$ & $22 \cdot 5$ \\
\hline 2nd measurement & $54 \cdot 0$ & $26 \cdot 3$ & $49 \cdot 3$ & $26 \cdot 5$ & $47 \cdot 4$ & $23 \cdot 1$ & $52 \cdot 3$ & $24 \cdot 9$ \\
\hline \multicolumn{9}{|l|}{ Placid } \\
\hline 1st measurement & $49 \cdot 9$ & 29.9 & $46 \cdot 0$ & $27 \cdot 1$ & $45 \cdot 3$ & $26 \cdot 3$ & $53 \cdot 7$ & $28 \cdot 7$ \\
\hline 2nd measurement & 51.9 & $30 \cdot 8$ & $49 \cdot 4$ & $28 \cdot 8$ & $46 \cdot 7$ & 28.5 & $52 \cdot 3$ & 31.4 \\
\hline \multicolumn{9}{|l|}{ Confident } \\
\hline 1st measurement & $65 \cdot 4$ & 23.9 & $64 \cdot 3$ & $27 \cdot 2$ & $69 \cdot 1$ & $23 \cdot 7$ & $68 \cdot 6$ & 22.9 \\
\hline 2nd measurement & $69 \cdot 3$ & $23 \cdot 1$ & $63 \cdot 3$ & $24 \cdot 1$ & $65 \cdot 8$ & $25 \cdot 1$ & $66 \cdot 5$ & $27 \cdot 1$ \\
\hline \multicolumn{9}{|l|}{ Tired } \\
\hline 1st measurement & $47 \cdot 3$ & $27 \cdot 6$ & $41 \cdot 2$ & $29 \cdot 3$ & 38.4 & $29 \cdot 4$ & $44 \cdot 2$ & $26 \cdot 6$ \\
\hline 2nd measurement & $42 \cdot 1$ & $26 \cdot 0$ & $40 \cdot 9$ & $25 \cdot 9$ & $39 \cdot 8$ & $26 \cdot 7$ & $38 \cdot 7$ & 28.9 \\
\hline \multicolumn{9}{|l|}{ Anxious } \\
\hline 1st measurement & $14 \cdot 0$ & $20 \cdot 3$ & $15 \cdot 9$ & $19 \cdot 6$ & $16 \cdot 6$ & $21 \cdot 7$ & $15 \cdot 0$ & 18.9 \\
\hline 2nd measurement & $17 \cdot 2$ & $25 \cdot 4$ & 11.9 & $15 \cdot 9$ & $12 \cdot 6$ & $17 \cdot 7$ & $10 \cdot 9$ & $13 \cdot 4$ \\
\hline \multicolumn{9}{|l|}{ Lively } \\
\hline 1st measurement & $51 \cdot 3$ & $26 \cdot 7$ & $45 \cdot 0$ & $30 \cdot 3$ & $50 \cdot 3$ & $25 \cdot 7$ & $53 \cdot 1$ & $22 \cdot 2$ \\
\hline 2nd measurement & $51 \cdot 2$ & $26 \cdot 9$ & 48.9 & $22 \cdot 9$ & $53 \cdot 3$ & $22 \cdot 8$ & $57 \cdot 0$ & $23 \cdot 1$ \\
\hline
\end{tabular}

GP/TP, given placebo/told placebo; GP/TG, given placebo/told glucose; GG/TG, given glucose/told glucose; GG/TP, given glucose/told placebo.

${ }^{*}$ For details of subjects and procedures, see pp. 174-175. 
glucose and expected glucose and sessions in which they received placebo but expected glucose $(P<0 \cdot 05)$. In addition, the post-drink levels of dejection were higher in the condition where subjects received glucose but expected placebo than when they received placebo and expected placebo $(P<0 \cdot 05)$.

There was an overall decrease in ratings of 'Energetic' over the course of each test session $(F(1,25) 5 \cdot 64$, $P=0 \cdot 026)$. There were no other significant main effects for this scale, or for any of the other rated moods $(P>0 \cdot 05)$.

\section{Relationships between task performance, glucose levels and mood state}

Correlational analyses were carried out between task performance during each session, glucose levels and change in glucose levels over the course of each session (with appropriate correction for multiple comparisons). There were no significant relationships between task performance and glucose levels $(P>0.05)$. Similarly, there were no significant relationships found between task performance and self-reported mood state $(P>0.05)$.

\section{Discussion}

There are a number of important findings of the present study. First, ingestion of a glucose drink improved performance on two of the tasks (recognition memory response times and Bakan task performance), but not on the other tasks in the assessment battery.

The finding that glucose improves performance on the Bakan task is inconsistent with the one other study which has used this task to investigate the effects of glucose on cognitive function (Benton et al. 1994). It is possible, however, that the differences in the results of the present study and those of Benton et al. (1994) can be accounted for in terms of methodological differences between the two studies. Specifically, subjects in the present study performed the Bakan task some 30 min post glucose ingestion. In the Benton et al. (1994) study, subjects in both the glucose and placebo conditions were given a $50 \mathrm{~g}$ glucose load $20 \mathrm{~min}$ prior to completing the task and were given either a further $25 \mathrm{mg}$ glucose or placebo (aspartame) immediately prior to the task. Given that plasma glucose levels are at maximum some 30 min after initial ingestion (Frayn et al. 1993), it may be concluded that at the time of Bakan task administration in the Benton et al. (1994) study, the glucose levels of subjects in both the glucose and placebo conditions were comparable.

The most important aspect of the results from subjects' Bakan task performance, however, is that the significant difference in performance between sessions in which subjects received glucose or aspartame was only true when they were told that they were given glucose. In the two conditions where subjects were informed that they were about to receive the aspartame drink, the actual content of the drink did not significantly affect performance. This finding indicates that there is at least some element of expectancy concerning the nature of the drink underlying performance on this task, although this result cannot be explained entirely in terms of expectancy effects. There are certain parallels between this finding and the results of other studies which have investigated placebo expectancy effects. Specifically, previous work has indicated that differences in performance resulting from ingestion of an active substance or placebo are also a function of the expectancy which subjects have concerning the active substance (Finnigan et al. 1995). The fact that there were no significant correlations between task performance and blood glucose levels also supports the conclusion that any improvements in Bakan task performance resulting from the ingestion of a drink are not entirely due to the glucose content of that drink.

Interpretation of the results of the recognition memory task is slightly more problematic. Although there was a glucose-related improvement in recognition times, there was no corresponding glucose-related improvement in the number of words correctly recognised. Thus, it is somewhat difficult to interpret this result in terms of glucose improving memory, especially given the failure to find glucose-related effects upon free recall performance (discussed later), although it supports the findings of Azari (1991), who also found that glucose administration did not affect recognition memory performance. The few published studies which have demonstrated improved recognition memory times have employed subjects with compromised brain function such as Alzheimer's dementia patients (e.g. Manning et al. 1993). Even in this case, however, there is some doubt regarding the reliability of this finding, since saccharin was used as a control substance and no data are reported concerning whether subjects could perceive differences in taste between the two drinks. It is also difficult to interpret the present finding that glucose administration improved recognition memory times in terms of enhanced motor performance, since glucose did not affect two-finger tapping performance. Further work needs to be carried out to elucidate the nature and replicability of this finding.

The present study also found that there was no significant effect either of the nature of the drink administered or expectancy upon verbal free recall. This finding stands in contrast to the results of a number of studies which have demonstrated significant improvements in performance amongst healthy adult subjects on this. It is, however, consistent with the larger body of evidence indicating that a glucose load exerts no beneficial effect on immediate verbal recall amongst healthy adult subjects (e.g. Azari, 1991; Benton \& Owens, 1993; Benton et al. 1994). Although it could be argued that the present failure to find a beneficial effect of glucose on the verbal free-recall task relates to the length of time between ingestion of the glucose and recall (e.g. Foster et al. 1998), this is unlikely given that subjects in the present study performed the recall task some $30 \mathrm{~min}$ after glucose ingestion. However, given the fact that Foster et al. (1998) and Manning et al. (1990) both used saccharin as a placebo control, it may be possible to explain those particular findings in terms of an expectancy effect. This explanation is made more likely by the fact that the studies mentioned earlier, which have failed to find effects of glucose on verbal recall performance, employed aspartame as a placebo control.

In conclusion, the present study found equivocal evidence for an improvement of performance resulting from the 
ingestion of a glucose drink in a healthy adult population when aspartame is used as a control substance. Although recognition memory times were improved as a result of glucose, this is difficult to explain either in terms of memory or motor performance. In addition, although performance on a rapid information processing (Bakan) task was improved as a result of glucose, this was only found to be the case under conditions where subjects were informed that they would be receiving glucose and not when they were informed that they would be receiving placebo. Taken as a whole, the present results offer little convincing or consistent support that glucose ingestion benefits cognition in a healthy adult, non food-deprived population.

\section{Acknowledgements}

This work was funded by the Biotechnology and Biological Sciences Research Council. The authors would like to thank David Lincoln for invaluable technical support during the course of this study and Paula Durlach for comments on an earlier draft of the manuscript.

\section{References}

Azari NP (1991) Effects of glucose on memory processes in young adults. Psychopharmacology 105, 521-524.

Bakan P (1959) Extroversion-introversion and improvement in an auditory vigilance task. British Journal of Psychology 50, 323-332.

Benton D, Brett V \& Brain PF (1987) Glucose improves attention and reaction to frustration in children. Biological Psychology 24, 95-100.

Benton D \& Owens DS (1993) Blood glucose and human memory. Biological Psychology 24, 95-100.

Benton D, Owens DS \& Parker PY (1994) Blood glucose influences memory and attention in young adults. Neuropsychologia 32, 595-607.

Benton D \& Sargent J (1992) Breakfast, blood glucose and memory. Biological Psychology 33, 207-210.

Brooke JD \& Toogoode S (1973) Factory accidents and carbohydrate supplements. Proceedings of the Nutrition Society 32, $94 \mathrm{~A}-95 \mathrm{~A}$.

Fillmore MT, Carscadden JL \& Vogel-Sprott M (1998) Alcohol, cognitive impairment and expectancies. Journal of Studies on Alcohol 59, 174-179.

Fillmore MT, Mulvihill LE \& Vogel-Sprott M (1994) The expected drug and its expected effect interact to determine placebo responses to alcohol and caffeine. Psychopharmacology 115, 383-388.

Fillmore M \& Vogel-Sprott M (1992) Expected effect of caffeine on motor performance predicts the type of response to placebo. Psychopharmacology 106, 209-214.

Finnigan F, Hammersley R \& Millar K (1995) The effects of expectancy and alcohol on cognitive-motor performance. Addiction 90, 661-672.

Foster JK, Lidder PG \& Sünram SI (1998) Glucose and memory: Fractionation of enhancement effects. Psychopharmacology 137, 259-270.

Frayn KN, Coppack GW, Humphreys SM, Clark ML \& Evans RD (1993) Periprandial regulation of lipid metabolism in insulintreated diabetes-mellitus. Metabolism - Clinical and Experimental, 42, 504-510.
Gold PE (1986) Glucose modulation of memory storage processing. Behavioural and Neural Biology 45, 342-349.

Green MW, Elliman NA \& Rogers PJ (1995) Lack of effect of short-term fasting on cognitive function. Journal of Psychiatric Research 29, 245-253.

Green MW, Elliman NA \& Rogers PJ (1997) The effects of food deprivation and incentive motivation on blood glucose levels and cognitive function. Psychopharmacology 134, 88-94.

Hall JL, Gonder-Frederick LA, Chewning WW, Silveira J \& Gold PE (1989) Glucose enhancement of performance on memory tests in young and aged humans. Neuropsychologia 22, $1129-1138$.

Hammersley R, Finnigan F \& Millar K (1998) Verbal expectancies and performance after alcohol. Addictive Behaviors 23, 489-496.

Kirsch I \& Weixel LJ (1988) Double blind versus deceptive administration of a placebo. Behavioural Neuroscience 102, 319-323.

Kvavilashvili L \& Ellis JA (1999) The effects of positive and negative placebos on human memory performances. Memory 7 , 421-437.

Johnson EO, Kamilaris TC, Chrousos GP \& Gold PE (1992) Mechanisms of stress: A dynamic overview of hormonal and behavioural homeostasis. Neuroscience and Biobehavioral Reviews 16, 115-130.

Lapp JE (1981) Effects of glycemic alterations and noun imagery on the learning of paired associates. Journal of Learning Disabilities 14, 35-38.

Manning CA, Hall JL \& Gold PE (1990) Glucose effects on memory and other neuropsychological tests in elderly humans. Psychological Science 1, 307-311.

Manning CA, Parsons MW, Cotter EM \& Gold PE (1997) Glucose effects on declarative and non-declarative memory in healthy elderly and young adults. Psychobiology 25, 103-108

Manning CA, Ragozzino ME \& Gold PE (1983) Glucose enhancement of memory in patients with probable senile dementia of the Alzheimer type. Neurobiology of Aging, 14, $523-528$.

Messier C, Desrochers A \& Gagnon M (1999) Effect of glucose, glucose regulation and word imagery value on human memory. Behavioural Neuroscience 113, 431-438.

Parsons MW \& Gold PE (1992) Glucose enhancement of memory in elderly humans: An inverted-U dose-response curve. Neurobiology of Aging 13, 401-404.

Peterson JB, Rothfleisch J, Zelazo PD \& Phil RO (1990) Acute alcohol intoxication and cognitive functioning. Journal of Studies on Alcohol 51, 114-122.

Pollitt E, Jacoby E \& Cueto S (1996) School breakfast and cognition among nutritionally at-risk children in the Peruvian Andes. Nutrition Reviews 54, S22-S26.

Pollitt E, Lewis NL, Garza C \& Schulman RJ (1983) Fasting and cognitive function. Journal of Psychiatric Research 17, $169-174$.

Pollitt E, Liebel RL \& Greenfield D (1981) Brief fasting, stress and cognition in children. American Journal of Clinical Nutrition 34, 1526-1533.

Rogers PJ \& Lloyd HM (1994) Nutrition and mental performance. Proceedings of the Nutrition Society 53, 443-456.

Sieber FE \& Traystmen RJ (1992) Special issues: Glucose and the brain. Critical Care Medicine 20, 104-114.

Sternberg S (1966) High speed scanning in human memory. Science 153, 652-654. 